\title{
L'objectif \\ "Zéro émissions nettes » de l'Accord de Paris : signification et implications
}

\author{
Quentin Perrier ${ }^{1}$, Céline Guivarch ${ }^{2}$, Olivier Boucher ${ }^{1}$ \\ 1. Institut Pierre-Simon Laplace, Sorbonne Université / CNRS, Paris \\ 2. Centre international de recherche sur l'environnement et le développement, \\ École des Ponts Paris Tech, Champs-sur-Marne
}

${ }^{*}$ Cet article est une version révisée et améliorée de la note interne IPSL-CMC ${ }^{\circ}$ 34, datée de mai 2018 et intitulée : "Zéro émissions nettes" : Signification et implications ».

perrier@centre-cired.fr

\section{Résumé}

L'Accord de Paris inclut l'objectif d'atteindre le «zéro émissions nettes " de gaz à effet de serre dans la seconde moitié du siècle. La formulation exacte de cet objectif soulève cependant plusieurs questions d'ordre scientifique. Comment distinguer les émissions et les absorptions anthropiques concernées par l'accord des flux naturels de ces mêmes gaz ? Quels gaz inclure dans l'accord, et comment comparer des gaz aux propriétés physiques différentes? Comment assurer la compatibilité du zéro émissions nettes avec les autres objectifs de l'Accord de Paris ? Cet article discute les choix possibles et leurs implications, afin de fournir des recommandations utiles pour préciser et rendre opérationnels les objectifs globaux de l'Accord de Paris.
- Quels gaz à effet de serre faut-il considérer?

'Accord de Paris s'est construit autour d'un objectif central, annoncé dès l'article 2 : limiter le réchauffement climatique à $2{ }^{\circ} \mathrm{C}$ et, si possible, à $1,5^{\circ} \mathrm{C}$. Cette finalité de long terme est assortie d'un jalon plus opérationnel dans l'article 4 : atteindre le «zéro émissions nettes » dans la seconde moitié du siècle. Autrement dit, il s'agit de tendre vers un équilibre entre les émissions anthropiques, en grande partie liées à l'utilisation de combustibles fossiles, et les absorptions anthropiques, via la plantation de forêts ou la captation et le stockage éventuel de $\mathrm{CO}_{2}$ atmosphérique.

Cependant, la formulation retenue pour cet article 4 interroge et son application nécessite de préciser plusieurs points, comme indiqué sur la figure 1 :

- Comment distinguer les flux anthropiques et les flux naturels ?

Comment définir un équilibre quand on considère plusieurs gaz?

- Comment définir qu'un équilibre est atteint quand on considère plusieurs gaz qui diffèrent par leurs durées de vie et leurs impacts sur le climat?

- Dans quelle mesure cet équilibre contribue-t-il à atteindre l'objectif de long terme de 1'article 2, à savoir contenir l'élévation de la température « nettement en-dessous de $2^{\circ} \mathrm{C}$ » et chercher à atteindre $1,5^{\circ} \mathrm{C}$ ?

À l'approche de la $24^{\mathrm{e}}$ Conférence des Parties (COP) de la Convention cadre des Nations unies sur le changement climatique (Ccnucc), les négociateurs sur le climat débattent du livre de règles (rulebook) qui doit préciser et rendre opérationnels les objectifs globaux de l'Accord de Paris. Cette étape est un prérequis indispensable pour définir précisément les efforts

Dans quelle mesure cet équilibre contribue-t-il à l'objectif de long terme de l'article 2 ?

Article 4 : En vue d'atteindre l'objectif de température à long terme énoncé à l'article 2, les Parties cherchent à parvenir [...] à un équilibre entre les émissions anthropiques par les sources et les absorptions anthropiques par les/puits de gaz à effet de serre au cours de la deuxiènne moitié du/siècle

Comment distinguer les flux anthropiques et les flux naturels?

Quels gaz faut-il considérer?

Figure 1. Questions soulevées par l'article 4 de l'Accord de Paris. 


\section{Abstract}

"Net zero emissions" objective in the Paris Agreement: Meaning and implications

The Paris Agreement includes the objective of achieving "net zero emissions" of greenhouse gases in the second half of the century. The exact wording, however, raises several scientific questions. How to distinguish between anthropogenic emissions and removals subject to the agreement, and the natural flows of these same gases? Which gases should be included in the agreement, and how to compare gases with different physical properties? How to ensure the compatibility of net zero emissions with the other objectives of the Paris Agreement? This article discusses possible choices and their implications, in order to provide useful recommendations for clarifying and operationalizing the global objectives of the Paris Agreement.

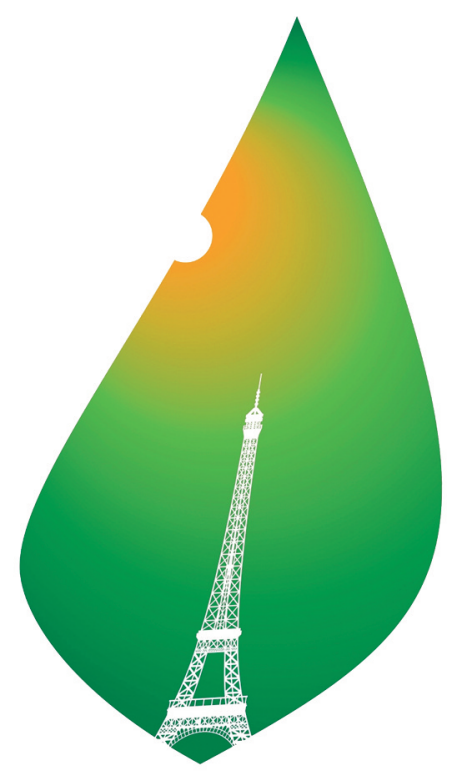

\section{COP21· CMP11}

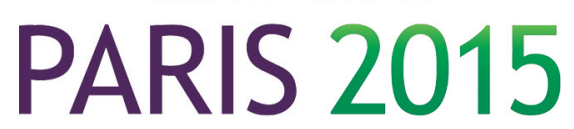

UN CLIMATE CHANGE CONFERENCE

Logo de la COP21 qui a donné lieu à l'Accord de Paris

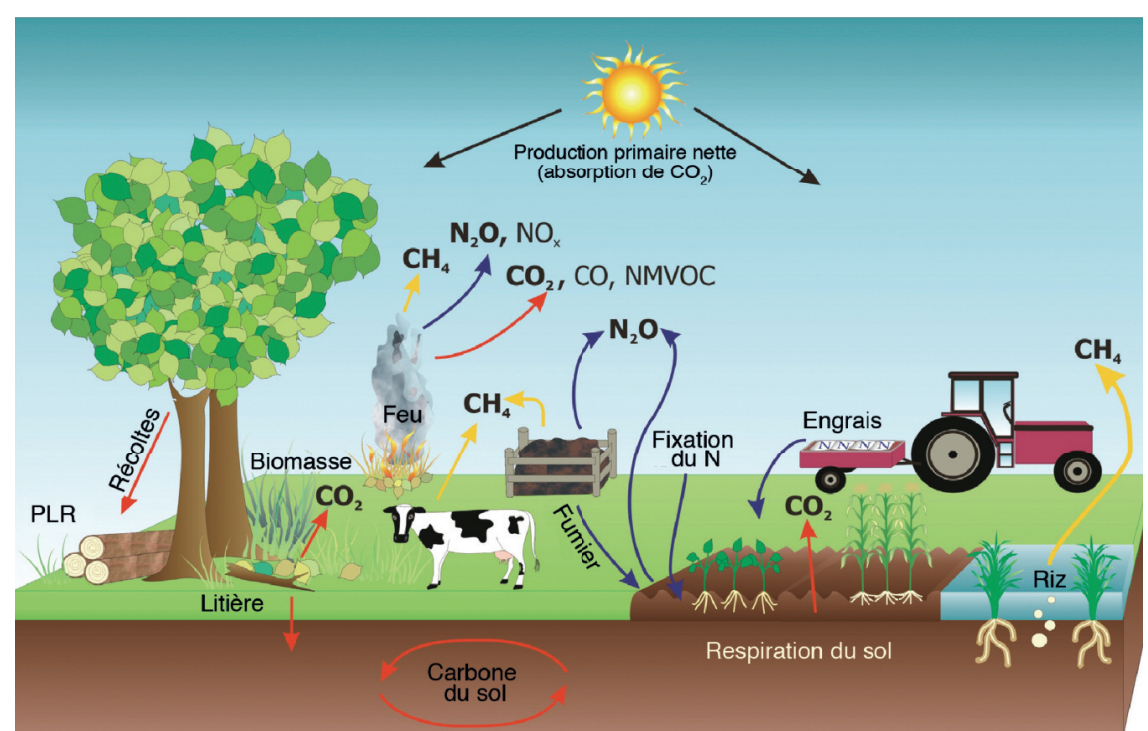

Figure 2. Représentation illustrée des émissions et absorptions dans le secteur de l'utilisation des terres. Source : IPCC (2006), avec traduction des auteurs.

respectifs des pays, et mettre en place les mécanismes de transparence et de suivi de ces efforts.

Dans ce contexte, ce travail discute les choix possibles face aux questions soulevées par la formulation de l'article 4. Nous étudions successivement : (1) la difficulté qui peut exister à distinguer flux anthropiques et naturels, (2) les gaz à considérer, (3) les options pour définir un équilibre multi-gaz et (4) la cohérence avec l'objectif de long terme.

\section{Distinguer les flux anthropiques des flux naturels}

Les émissions industrielles et celles liées à l'utilisation de combustibles fossiles sont clairement identifiées comme anthropiques, et il existe des règles précises proposées par le Giec pour en dresser les inventaires (IPCC, 2006). A contrario, il y a consensus pour considérer les absorptions de $\mathrm{CO}_{2}$ par les océans et les forêts primaires comme étant naturelles, même si celles-ci sont indirectement dues à l'augmentation de la concentration atmosphérique de $\mathrm{CO}_{2}$ qui, elle, est bien d'origine anthropique. En revanche, il peut être difficile, voire impossible, de faire la part du naturel et de l'anthropique dans le secteur de l'utilisation des terres et des forêts ${ }^{1}$. En effet, ce secteur se distingue par le fait qu'il contient à la fois de nombreux puits et de nombreuses sources, comme le montre la figure 2. Ces puits et sources peuvent aussi dépendre de manière importante de phénomènes dits naturels (sécheresses, inondations, feux...) mais dont l'origine est parfois indirectement attribuable à l'activité humaine. Ainsi, un feu de forêt peut avoir été déclenché volontairement ou non par une personne. Le changement climatique, d'origine anthropique, accentue aussi les sécheresses et la probabilité d'occurrence de feux dans certaines régions.

Pour faire face à cette difficulté, la Ccnucc a adopté le concept de « terre gérée » : sont considérées comme anthropiques toutes les émissions et absorptions ayant lieu sur des terres déclarées comme "gérées 》 par les États (Iversen et al., 2014). Cette définition permet notamment de définir comme anthropiques l'ensemble des absorptions de $\mathrm{CO}_{2}$ par les forêts gérées, bien que celles-ci soient stricto sensu en partie naturelles et considérées comme telles dans les « budgets carbone » estimés par le Groupe intergouvernemental d'experts sur l'évolution du climat (Giec). Sans être entièrement satisfaisante d'un point de vue théorique, cette définition pragmatique offre un cadre opérationnel aux Parties de l'Accord de Paris pour mesurer leurs émissions et les déclarer à la Ccnucc. Les « technologies à émissions négatives » qui consistent à retirer $\mathrm{du} \mathrm{CO}_{2}$ de l'atmosphère, si elles se concrétisent, devront aussi être quantifiées et prises

1. Plus précisément, on parle ici du secteur qui comprend l'utilisation des terres, le changement d'affectation des terres et la foresterie (UTCATF, ou LULUCF en anglais). 
en compte comme puits anthropiques. On pense là par exemple à l'utilisation de biomasse comme source d'énergie couplée avec une technologie de captation et de stockage du $\mathrm{CO}_{2}$ émis lors de la combustion ${ }^{2}$, voire à de la captation directe ${ }^{3}$ du $\mathrm{CO}_{2}$ atmosphérique par des méthodes chimiques et à son stockage.

\section{Les aérosols, agents climatiques sous-estimés}

À long terme, la température de la planète est surtout influencée par l'accumulation dans l'atmosphère de gaz à effet de serre (GES) à longue durée de vie. Le protocole de Kyoto portait ainsi sur six gaz ou familles de gaz à effet de serre : le dioxyde de carbone $\left(\mathrm{CO}_{2}\right)$, le méthane $\left(\mathrm{CH}_{4}\right)$, le protoxyde d'azote $\left(\mathrm{N}_{2} \mathrm{O}\right)$, l'hexafluorure de soufre $\left(\mathrm{SF}_{6}\right)$, les hydrofluorocarbures (HFC) et les perfluorocarbures (PFC). Lors de la COP de Doha, en 2012, le protocole de Kyoto a été prolongé jusqu'en 2020 et le trifluorure d'azote $\left(\mathrm{NF}_{3}\right)$ a été ajouté à la liste des gaz concernés par cet accord, aussi appelé « Kyoto II ». Les chlorofluorocarbures (CFC) et hydrochlorofluorocarbures (HCFC) sont quant à eux couverts par le protocole de Montréal et ses amendements, qui vise à les éliminer (Boucher, 2017). Il n'y a donc pas forcément lieu de les considérer dans le cadre de l'Accord de Paris.

Ce choix de gaz à effet de serre exclut cependant d'autres composés à courte durée de vie dans l'atmosphère qui influencent aussi la température de la planète, comme l'ozone, le carbone suie et autres aérosols (c'est-à-dire des particules en suspension dans l'air). Dans leur ensemble, les aérosols d'origine anthropique contribuent à refroidir le climat; cependant, en concentrations trop élevées, ils sont nocifs pour la santé, notamment pour les systèmes respiratoires et cardiovasculaires. Les politiques d'amélioration de la qualité de l'air continuent de se renforcer dans les pays développés et commencent à être mises en place dans les pays en voie de développement. Une diminution des émissions d'aérosols pourrait entraîner un réchauffement pouvant aller jusqu'à 0,5 ou $1^{\circ} \mathrm{C}$, avec des effets plus marqués dans les zones fortement peuplées (Samset et al., 2018). Il convient donc d'inclure les aérosols dans la réflexion, notamment pour anticiper le risque d'une augmentation trop forte de la température.

Par ailleurs, l'équilibre entre émissions et absorptions anthropiques ne conduit pas instantanément à une stabilisation de la température, car le système climatique possède une inertie importante. L'équilibre mentionné dans l'article 4 de l'Accord de Paris ne porte que sur les émissions et absorptions anthropiques. Une fois cet équilibre atteint, le cycle naturel du carbone et le climat de la Terre finiront eux aussi par s'équilibrer, mais après une phase transitoire très longue, de plusieurs siècles, voire de plusieurs millénaires. Plus précisément, si on retourne à un équilibre entre puits et sources anthropiques de $\mathrm{CO}_{2}$, les puits naturels resteront un peu plus forts pendant quelque temps du fait des concentrations élevées de $\mathrm{CO}_{2}$ dans l'atmosphère, ce qui contribuera à diminuer les concentrations de $\mathrm{CO}_{2}$. En revanche, l'effet inertiel du système climatique conduira à un réchauffement. Compte tenu des incertitudes sur les processus concernés, l'effet net de cette période transitoire pourrait donc être un réchauffement ou un refroidissement.

\section{Définir un équilibre multi-gaz}

Les différents gaz à effet de serre présentent des potentiels d'atténuation différents. En particulier, les émissions de $\mathrm{CH}_{4}$ et de $\mathrm{N}_{2} \mathrm{O}$ sont en partie liées à l'agriculture et pourraient être difficiles à éliminer complètement. À l'inverse, il est envisageable qu' on puisse non seulement réduire les émissions de $\mathrm{CO}_{2}$ mais aussi capter du $\mathrm{CO}_{2}$ atmosphérique, ce qui correspondrait à des « émissions négatives ». Bien que les technologies de captation n'aient pas encore atteint la maturité industrielle, leur déploiement à moyen terme correspond aux trajectoires proposées par les modèles économie-climat qui cherchent à respecter l'objectif des $2^{\circ} \mathrm{C}$. On imagine donc que les émissions résiduelles de certains gaz à effet de serre, notamment le $\mathrm{CH}_{4}$ et le $\mathrm{N}_{2} \mathrm{O}$, pourront être compensées par la captation de $\mathrm{CO}_{2}$.

Cependant, les différents gaz à effet de serre ont des propriétés physiques très variables, notamment en termes de durée de vie et d'efficacité radiative ${ }^{4}$. Par exemple, le méthane a une durée de vie bien plus courte que le $\mathrm{CO}_{2}$, mais une efficacité radiative bien plus importante. Quelle serait la quantité de $\mathrm{CO}_{2}$ à capter pour compenser l'émission d'une tonne de méthane ? Pour rendre comparable l'impact des différents gaz sur le climat, on définit généralement un " équivalent $\mathrm{CO}_{2}$ " . Autrement dit, on attribue à chaque gaz un coefficient qui permet de comparer l'émission de ce gaz à l'émission d'une même masse de $\mathrm{CO}_{2}$, le $\mathrm{CO}_{2}$ étant choisi comme référence. Ces équivalences sont calculées au moyen de modèles numériques de transfert radiatif qui représentent l'effet de ces gaz dans l'atmosphère.

Il n'existe pourtant pas une manière unique de définir ces coefficients. Les valeurs vont dépendre d'un choix, en partie arbitraire, sur la façon de comparer ces gaz. Souhaite-t-on plutôt comparer les effets sur le climat à court terme ou à long terme ? L'effet cumulé ou l'effet à une date donnée ? Ces choix aboutissent à définir une "métrique " d'équivalence pour comparer les différents gaz à effet de serre. Plusieurs métriques existent aujourd'hui, mais aucune n'est parfaite. Le choix d'une métrique est associé à un jugement de valeur sur l'échelle de temps associé au changement climatique (Boucher, 2010). Selon la métrique retenue, le coefficient d'équivalence entre les différents gaz peut être très différent. Pour le méthane, ce coefficient peut ainsi varier d'un facteur 20, comme indiqué dans le tableau 1.

La Ccnucc et le Giec ont jusqu'à aujourd'hui privilégié la métrique GWP 100 , qui compare le forçage radiatif cumulé sur 100 ans d'un gaz et de la même masse de $\mathrm{CO}_{2}$ émis à un instant donné ${ }^{5}$. L'utilisation d'une métrique avec

2. Bio-energy with carbon capture and storage ou BECCS

3. Les technologies de captation directe visent à capter le $\mathrm{CO}_{2}$ directement dans l'atmosphère. Elles sont à distinguer des technologies de captation plus classiques (carbon capture and storage ou CCS) qui visent à capter et stocker le $\mathrm{CO}_{2}$ émis par la combustion de combustibles fossiles, par exemple à la sortie d'une centrale thermique. Le CCS permet de réduire les émissions mais n'induit pas d'émissions négatives.

4. L'efficacité radiative d'un gaz à effet de serre correspond à la capacité de ce gaz à modifier le bilan radiatif de la planète. Elle s'exprime en $W^{-2}$ par unité de rapport de mélange dans l'atmosphère (partie par million, ppm, ou partie par milliars, $p p b$ ). 
Tableau 1. Équivalent $\mathrm{CO}_{2}$ de différents gaz selon diverses métriques. Source : IPCC (2014).

\begin{tabular}{lccccc}
\hline Gaz & $\begin{array}{c}\text { Durée de vie } \\
\text { (années) }\end{array}$ & GWP 20 & GWP 100 & GTP 20 & GTP 100 \\
\hline Dioxyde de carbone $\left(\mathrm{CO}_{2}\right)$ & $*$ & 1 & 1 & 1 & 1 \\
\hline Méthane $\left(\mathrm{CH}_{4}\right)$ & 12,4 & 84 & 28 & 67 & 4 \\
\hline Protoxyde d'azote $\left(\mathrm{N}_{2} \mathrm{O}\right)$ & 121 & 264 & 265 & 277 & 234 \\
\hline HFC-152a & 1,5 & 506 & 138 & 174 & 19 \\
\hline
\end{tabular}

* Pour le $\mathrm{CO}_{2}$, il n'est pas possible d'indiquer une durée de vie unique, car sa décroissance ne suit pas une loi exponentielle simple. Entre un tiers et la moitié du $\mathrm{CO}_{2}$ est absorbé au bout de quelques décennies ; 15 à $40 \%$ peuvent rester plusieurs milliers d'années dans l'atmosphère.

un horizon plus proche (par exemple 20 ans avec les métriques GWP 20 et GTP 20) permet de mieux considérer les effets de réchauffement à court terme. En revanche, ces métriques à 20 ans ne sont pas adaptées pour fournir une équivalence quant à l'évolution à long terme de la température, car elles ne prennent pas en compte l'effet d'accumulation des gaz à longue durée de vie dans l'atmosphère.

Pour atteindre l'équilibre, les contributions relatives des différents secteurs économiques (industrie, énergie, agriculture) seront donc différentes selon la métrique retenue, car les proportions relatives des différents gaz émis sont très différentes. Au niveau international, ceci peut également affecter les efforts relatifs des États, selon la structure de leur économie et de leurs émissions.

En outre, le choix d'une métrique influence l'impact d'un équilibre sur la température de long terme. L'objectif d'un équilibre entre émissions et absorptions est souvent présenté comme une façon de stabiliser la température à long terme. Or, cette équivalence « intuitive » n'est pas nécessairement vérifiée : avec certaines métriques d'équivalent $\mathrm{CO}_{2}$, l'équilibre peut aboutir à un réchauffement ou un refroidissement du climat à long terme. Par exemple, une métrique donnant un poids important aux gaz à courte durée de vie aboutirait à compenser des émissions de méthane en captant de grandes quantités de $\mathrm{CO}_{2}$, faisant ainsi baisser la température à long terme. Ainsi, avec la métrique GWP 100, un équilibre entre émissions et absorptions conduirait à un léger refroidissement sur le long terme, dans l'hypothèse probable où l'on compense des émissions de méthane par des absorptions de $\mathrm{CO}_{2}$.

Une autre métrique, appelée GWP* (qui assimile une émission pérenne d'une espèce à courte durée de vie comme le méthane à une émission instantanée de $\mathrm{CO}_{2}$ ) offre une meilleure adéquation entre les deux objectifs d'équilibre des émissions et de stabilisation des températures (Fuglestvedt et al., 2017). Proposée récemment, cette métrique n'a cependant pas encore d'assise politique. À l'inverse, le GWP 100, déjà utilisé par le Giec et la Ccnucc, bénéficie d'une plus large notoriété et d'un consensus entre les États.

Une autre option serait d'exclure le méthane, dont la durée de vie est sensiblement plus courte que le $\mathrm{CO}_{2}$, voire de ne considérer que le $\mathrm{CO}_{2}$, pour que l'équilibre conduise bien à une stabilisation des températures à long terme (Tanaka et $\mathrm{O}^{\prime}$ Neill, 2018). Cependant, exclure certains gaz de l'Accord de Paris peut aboutir à des pics de température plus élevés à court et moyen terme et à des politiques d'atténuation sousoptimales.

\section{Un potentiel limité pour les émissions négatives}

L'équilibre entre émissions et absorptions de gaz à effet de serre peut être suffisant pour atteindre les objectifs de long terme de l'article 2 si la diminution des émissions débute prochainement. Les émissions de certains secteurs étant très difficiles à réduire, par exemple dans l'agriculture, le «zéro émissions nettes " sera la résultante d'émissions faibles, compensées par des absorptions elles aussi relativement faibles (figure 3a).

Cependant, les objectifs affichés par les États dans le cadre de l'Accord de Paris nous placent plutôt sur une trajectoire de pic tardif, avec un dépassement des $2{ }^{\circ} \mathrm{C}$ à moyen terme (Benveniste et al., 2018). Dans ce cas, l'équilibre n'est plus suffisant : des émissions nettes négatives seront nécessaires pour atteindre l'objectif de $2{ }^{\circ} \mathrm{C}$ à long terme. Ces émissions nettes négatives impliquent des absorptions de grande ampleur dans les scénarios envisagés (figure $3 b$ ).

La faisabilité technique, économique et politique des émissions négatives ${ }^{6}$ à grande échelle n'est cependant pas assurée. Quel est leur potentiel réel,

5. Le GWP (Global Warming Potential ou pouvoir de réchauffement global) mesure le forçage radiatif cumulé d'un kilogramme du gaz concerné, rapporté à la même quantité d'un kilogramme de $\mathrm{CO}_{2}$ cumulé sur l'ensemble de la période considérée (par exemple, sur 100 ans pour le GWP 100). Le GTP (Global Temperature change Potential) donne l'augmentation de température globale à un instant précis (par exemple, au bout de 100 ans pour le GTP 100) en réponse à une émission ponctuelle d'un gaz, par rapport à l'émission ponctuelle d'une même masse de $\mathrm{CO}_{2}$. 6. Il convient de distinguer les émissions nettes négatives (c'est-à-dire émissions moins absorptions) d'une part et les émissions négatives (c'est-à-dire les absorptions uniquement) d'autre part.

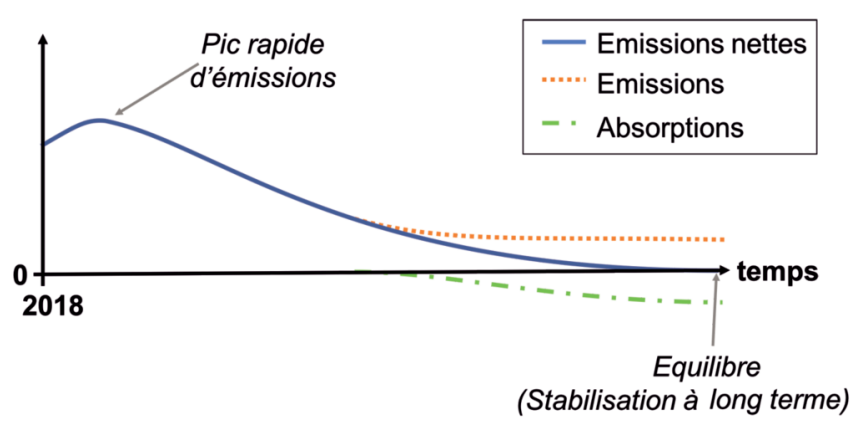

(a) Trajectoire avec pic rapide des émissions et atteinte de l'équilibre sans dépasser $2^{\circ} \mathrm{C}$

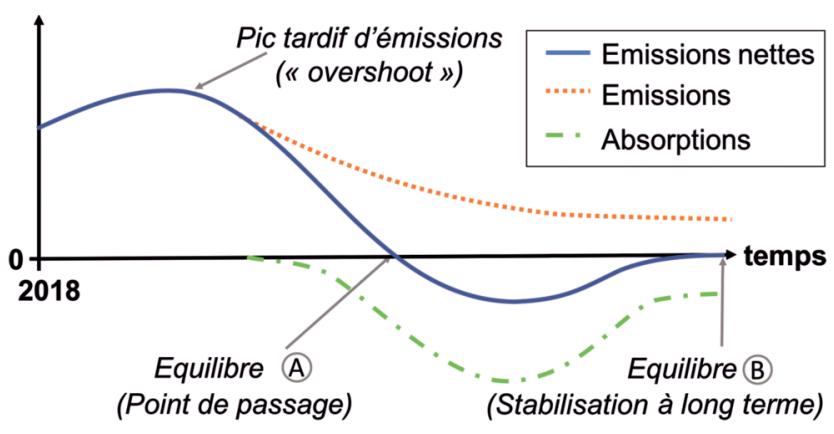

(b) Trajectoire avec pic tardif d'émissions et dépassement temporaire possible des $2{ }^{\circ} \mathrm{C}$ (overshoot)

Figure 3. Trajectoires stylisées d'émissions nettes tendant vers zéro, avec pic rapide ou tardif des émissions. 
dans un monde plus chaud et plus peuplé, davantage soumis aux problèmes de sécheresse et de compétition des sols pour l'agriculture (van Vuuren et al., 2017) ? Comment mettre en place un système des échanges d'émissions au niveau international, au sein d'un processus reconnu des États ? Ces incertitudes impliquent que tout retard dans l'action est un pari risqué, aux dépens des générations futures qui devront supporter l'essentiel des dommages climatiques - dont le coût est certes difficile à estimer précisément, mais certainement considérable (Hallegatte, 2006). Les techniques de géo-ingénierie pourraient alors devenir le dernier recours pour contenir l'élévation des températures, mais ces options présentent des incertitudes techniques et de nombreux risques pour le climat (Boucher, 2012). L'équilibre (point de passage A dans la figure $3 \mathrm{~b}$ ) doit donc être atteint le plus tôt possible pour augmenter les chances d'atteindre les objectifs de long terme de l'article 2 .

\section{Conclusions}

L'article 4 de l'Accord de Paris, qui introduit la notion de «zéro émissions nettes ", nécessite plusieurs précisions avant de pouvoir devenir opérationnel. En analysant les questions soulevées par la formulation actuelle, nous aboutissons aux quatre conclusions suivantes :

- pour distinguer sources anthropiques et naturelles, la Convention cadre des
Nations unies sur les changements climatiques fournit une base imparfaite mais utile en pratique ;

- les gaz retenus peuvent être ceux de Kyoto II, c'est-à-dire ceux à longue durée de vie, mais les effets des aérosols et l'inertie du système climatique ne doivent pas être négligés ;

- le choix d'un équivalent $\mathrm{CO}_{2}$ influera sur les efforts relatifs de réduction d'émissions entre pays et entre secteurs économiques, mais aussi sur la compatibilité avec l'article 2. La métrique GWP 100, déjà utilisée par la Ccnucc, offre un compromis intéressant : elle permet de prendre en compte l'inertie de long terme de certains composés, tout en intégrant les phénomènes de court terme. Cependant, avec cette métrique, l'équilibre en sources et puits anthropiques pourrait aboutir à un léger refroidissement de la température de la planète à long terme. Une autre métrique, le GWP*, offre une meilleure adéquation entre équilibre des émissions et stabilisation de la température ;

- pour assurer la cohérence avec l'article 2, l'équilibre pourrait ne pas être suffisant, surtout s'il est atteint trop tard. Dans ce cas, des « émissions négatives 》 importantes seront nécessaires, mais leur potentiel limité implique de réduire le plus rapidement possible les émissions pour espérer respecter l'objectif de $2{ }^{\circ} \mathrm{C}$.

Expliciter l'objectif de « zéro émissions nettes » à l'échelle globale est un jalon nécessaire avant de décliner un tel objectif au niveau de pays, de régions, de villes ou d'entreprises. Cette descente d'échelle pose cependant d'autres questions, en particulier sur le périmètre des émissions et des absorptions à considérer et sur la répartition des efforts. Faut-il comptabiliser uniquement les émissions intérieures au territoire d'un pays, d'une région ou d'une ville, ou inclure également les émissions importées et exportées ? Comment répartir les efforts d'émissions négatives à l'échelle mondiale de façon efficace et équitable, sachant que le potentiel mondial est limité et varie selon les régions et les acteurs ? De même, dans le cas d'une entreprise, faut-il inclure ou non les émissions qui ont lieu en amont (dues aux achats de l'entreprise) ou en aval (en lien avec l'utilisation des produits vendus) ? Où s'arrête la responsabilité d'un territoire ou d'une entreprise et où commence celle du citoyen ? Comment quantifier et sommer les contributions d'acteurs non étatiques dont les périmètres se recoupent ? Voilà autant de questions pour lesquelles la science doit guider les choix méthodologiques et aider à apporter des réponses.

\section{Remerciements}

Les auteurs remercient Amélie Rajaud, François-Marie Bréon et Anne Guillaume pour leurs commentaires sur les précédentes versions du manuscrit. Ce travail a bénéficié d'une aide du ministère de la Transition écologique et solidaire au titre de la Convention relative à l'attribution d'un appui financier au bénéfice des services climatiques.

\section{Bibliographie}

Benveniste H., Boucher 0., Guivarch C., Le Treut H., Criqui P., 2018. Impacts of nationally determined contributions on 2030 global greenhouse gas emissions: uncertainty analysis and distribution of emissions. Environ. Res. Lett., 13, 014022. doi: 10.1088/1748-9326/aaa0b9

Boucher 0., 2010. Quel rôle pour les réductions d'émission de méthane dans la lutte contre le changement climatique ? La Météorologie, 68, 35-40. doi: 10.4267/2042/31994

Boucher 0., 2012. L'ingénierie climatique face au réchauffement climatique : solution d'avenir ou fuite en avant ? La Météorologie, 78, 31-43. doi: 10.4267/2042/47513

Boucher 0., 2017. L'Amendement de Kigali au Protocole de Montréal vise l'élimination des hydrofluorocarbures. La Météorologie, 96, 3-5. doi: 10.4267/2042/61963

Fuglestvedt J., Rogelj J., Millar R., Allen M., Boucher 0., Forster P., Kriegler E., Shindell D., 2017. Implications of possible interpretations of "greenhouse gas balance" in the Paris Agreement. Philos. Trans. R. Soc. A Math. Phys. Eng. Sci., 376. doi: 10.1098/rsta.2016.0445

Hallegatte S., 2006. L'évaluation économique des dommages du changement climatique. La Météorologie, 52, 38-47. doi: 10.4267/2042/20054

IPCC, 2006. 2006 IPCC Guidelines for National Greenhouse Gas Inventories. Prepared by the National Greenhouse Gas Inventories Programme. IGES, Japan.

IPCC, 2014. Climate Change 2014: Synthesis Report. Contribution of Working Groups I, II and III to the Fifth Assessment Report of the Intergovernmental Panel on Climate Change. IPCC, Geneva, Switzerland, $151 \mathrm{p}$

Iversen P., Lee D., Rocha M., 2014. Comprendre l'utilisation des terres dans la CCNUCC. Forest Carbon, Markets and Communities

Samset B.H., Sand M., Smith C.J., Bauer S.E., Forster P.M., Fuglestvedt J.S., Osprey S., Schleussner C.F., 2018. Climate impacts from a removal of anthropogenic aerosol emissions. Geophys. Res. Lett., 45, 1020-1029. doi: 10.1002/2017GL076079

Tanaka K., O'Neill B.C., 2018. The Paris Agreement zero-emissions goal is not always consistent with the $1.5^{\circ} \mathrm{C}$ and $2^{\circ} \mathrm{C}$ temperature targets. Nat. Clim. Change, 8 , $319-324$. doi: 10.1038/s41558-018-0097-x 